Pacific Journal of Mathematic 


\title{
INFINITE GAMES AND SPECIAL BAIRE SPACE EXTENSIONS
}

\author{
M. R. KROM
}

\begin{abstract}
Two known characterizations of Baire spaces, one in terms of open covers and one in terms of infinite games, are proved directly to be equivalent. It is shown that any topological space is a dense subspace of some $\alpha$-favorable space (a special kind of Baire space) and that any space which is normal and regular (but not necessarily $T_{1}$ ) is a dense subspace of some regular $\alpha$-favorable space.
\end{abstract}

1. Baire space characterizations. For any topological space $X$ let $G(X)$ be the infinite two person game in which players $\alpha$ and $\beta$ take turns choosing the terms of a countably infinite nested sequence of nonempty open sets, in which $\beta$ chooses the first term of the seqence, and in which $\alpha$ wins a play if and only if the intersection of the sequence formed is nonempty [3, p. 115]. A stratery for a game $G(X)$ is a function $f$ from the set $\tau^{*}$ of nonempty open sets of $X$ such that for $U \in \tau^{*}, f(U) \in \tau^{*}$ and $f(U) \subset U$ (a strategy is a rule for determining a player's next move in terms of just the preceding move). When $\beta$ uses strategy $f$ in $G(X)$ his first move will be $f(X)$. A strategy is a winning strategy for a player in case it produces a win for that player when played against any strategy for the opponent. A topological space $X$ is called $\alpha$-favorable in case $\alpha$ has a winning strategy, $\beta$-favorable in case $\beta$ has a winning strategy, and indeterminate in case neither player has a winning strategy in $G(X)$. Our first theorem follows from properties of the Banach-Mazur Game [11, Chapter 6] and is an immediate conseqence of Theorem 2 in [7]. A Baire space is a topological space $X$ such that the intersection of countably many dense open subsets of $X$ is a dense subset of $X[9$, p. 268].

THEOREM 1. A topological space is $\beta$-favorable if and only if it is not a Baire space.

Our direct demonstration of the equivalence of two characterizations of Bairespaces is presented in the form of an alternative proof of McCoy's Theorem 1 in [8]. (It is known that "countable" may be omitted from the statement of this theorem, see [9] and [4].) The proof also shows that whenever there is a point finite open cover which is not locally finite on a dense set of points then there is a countable one of the special form that we construct. For any 
nonempty open set $U$ of a topological space $X$ and any strategy $f$ for $G(X)$ the $f$-subsets of $U$ consist of just $f(U)$ if $U$-cl $(f(U))$ is empty and the two open subsets $f(U)$ and $U-\mathrm{cl}(f(U))$ otherwise.

Theorem 2 (McCoy). A topological space $X$ is a Baire space if and only if every point finite open cover of $X$ is locally finite at a dense set of points.

Game-theoretic proof. First assume that $X$ is not a Baire space. Then, by Theorem 1 , there is a winning strategy $f$ for $\beta$ in $G(X)$. Let $\omega$ be the set of nonnegative integers, let $S_{0}=\{f(X)\}$, for $n \in \omega$ let $S_{n+1}$ be the set of $f$-subsets of members of $S_{n}$, and let $S=\mathbf{U}_{n \in \omega} S_{n}$. Then $S \cup\{X\}$ is a point finite countable open cover of $X$ which is not locally finite on any dense set of points. The open cover $S \cup\{X\}$ is point finite because any two sets in $S$ with nonempty intersection must be nested and any countable family of sets in $S$ with nonempty intersection would, together with $f(X)$, form a play in $G(X)$ in which $\beta$ uses strategy $f$. Such a play would contradict the assumption that $f$ is a winning strategy for $\beta$. By construction $S$ is not locally finite at any point of the nonempty open set $f(X)$.

Next assume that $\mathscr{C}=\left\{U_{i} \mid i \in \gamma\right\}$ is a point finite open cover of $X$ which is not locally finite on any dense set of points, where $\gamma$ is some ordinal and $U_{i} \neq U_{j}$ for $i \neq j$. We describe a winning strategy $f$ for $\beta$ in $G(X)$. The set of points at which $\mathscr{C}$ is locally finite is not dense in $X$. Let $f(X)$ be any nonempty open set on which $\mathscr{C}$ is not locally finite such that $f(X) \neq X$. For any nonempty open $V \subset f(X)$ let $f(V)=V \cap U_{k}$ where $k$ is the least ordinal in $\gamma$ such that $\phi \neq V \cap U_{k} \neq V$. For nonempty $U \not \subset f(X)$ let $f(U)=U$. The function $f$ is defined for all nonempty open sets because $\mathscr{C}$ is not locally finite but is point finite at every point of $f(X)$ and $f$ is a winning strategy for $\beta$ because $\mathscr{C}$ is point finite. By Theorem $1, X$ is not a Baire space.

2. Special Baire space extensions. We will use open filters to form extensions in the same way as is done by R. A. McCoy [8, p. 201], but the game-theoretic characterization of Baire spaces shows that a more restricted class of filters will suffice and that the resulting extensions are a special kind of Baire space. For any topological space $X$, any set $F$ of open filters on $X$, and any open $U$ in $X$, let $U^{*}=U \cup\{\mathscr{F} \in F \mid U \in \mathscr{F}\}$. Let $X_{F}$ be the disjoint union of $X$ and $F$ with topology generated by the base $\left\{U^{*} \mid U\right.$ is open in $X$ \}. Hereafter we will denote this base for the topology of $X_{F}$ by $\mathscr{B}(X, F)$. A space $X$ is always a dense subspace of $X_{F}$ and in [8] it is shown that if $F$ includes all free open ultrafilters on $X$ 
then $X_{F}$ is a Baire space. The stronger statement is true that if $F$ includes all free open ultrafilters then $X_{F}$ is $\alpha$-favorable; any strategy for $\alpha$ in $G\left(X_{F}\right)$ is a winning strategy in this case because there are no winning plays for $\beta$ (there are no countably infinite nested sequences of nonempty open sets with empty intersection).

One point of significance in the stronger conclusion is that the class of $\alpha$-favorable spaces is closed under topological products while the larger class of Baire spaces is not. Also from Theorem 1 above, it is easy to see that for any $\alpha$-favorable space $X$ and any Baire space $Y$ the topological product $X \times Y$ is a Baire space $(\mathrm{H}$. E. White observed that this is true for weakly $\alpha$-favorable spaces $X$ [14], cf. also the theorems on pages 3 and 9 of [1] and Theorem 2, page 158 of [10]). However, assuming the continuum hypothesis (or Martin's Axiom [13]), there is a Baire space $X$ such that $X \times X$ is not a Baire space [10]. For such a Baire space $X$ the game $G(X)$ is indeterminate.

The inclusion of all free open ultrafilters in $F$ to form the Baire space extension is generally extravagant. From Theorem 1 above it follows that if $F$ is any family of open filters on $X$ then $X_{F}$ is a Baire space if and only if for any winning strategy $f$ for $\beta$ in $G(X)$ there is an $\mathscr{F} \in F$ such that $\mathscr{F}$ contains the terms of some play in which $f$ is used by $\beta$ (see the proof of Theorem 3 below for further details). In particular if $X$ is quasi-regular (each nonempty open set includes the closure of a nonempty open set $[1]$, p. 1) we need only use open filters in $F$ that are generated by filter bases consisting of the terms of plays in which $\alpha$ uses quasiregularity with each move. For example, let $Q$ be the space of rational numbers with the usual topology and let $F(R)$ be a set of free open filters on $Q$ consisting of one filter $\mathscr{F}_{r}$ for each irrational real number $r$ and such that

$$
\left\{x \in Q \mid r-\frac{1}{n+1}<x<r+\frac{1}{n+1}\right\} \in \mathscr{F}_{r}
$$

for all $n \in \omega$. Then $Q_{F(R)}$ is a regular Baire space extension of $Q$. But if $F$ is the set of all free open ultrafilters on $Q$ then the Baire space extension $Q_{F}$ is not regular. In particular there is a filter $\mathscr{F}^{0} \in F$ such that

$$
\left\{x \in Q \mid 0<x<\frac{1}{n+1}\right\} \in \mathscr{F}^{0}
$$

for all $n \in \omega$ and the closure of any open set in $Q_{F}$ which contains the point $\mathscr{F}^{0}$ is not disjoint from the one point closed set $\{0\}$. A regular filter on a topological space is a filter $\mathscr{F}$ of open sets such 
that for any $U \in \mathscr{F}$, there exists $V \in \mathscr{F}$ such that $\bar{V} \subset U[9$, p. 268 and 4, p. 329]. By Zorn's lemma, any set $\mathscr{H}$ of nonempty open sets in a topological space is included in a maximal free regular filter if $\mathscr{H}$ has the finite intersection property, empty intersection, and for any $M \in \mathscr{H}$ there is an $N \in \mathscr{H}$ such that $\bar{N} \subset M$. In particular the set of terms of a winning play for $\beta$ in a game $G(X)$ in which $\alpha$ uses quasi-regularity with each move is included in a maximal free regular filter. A regular filter $\mathscr{F}$ on a topological space $X$ is a regular end in case for any two open subsets $U, V$ of $X$ such that $\bar{U} \cap \bar{V}=\phi$, if $W \cap U \neq \phi$ for all $W \in \mathscr{F}$ then $X-\bar{V} \in \mathscr{F}[12, \mathrm{p}$. 337]. It is known that if $X$ is a regular space and $F$ is the set of all regular ends on $X$ then $X_{F}$ is regular [12, Theorem 4.6]. In a similar way, and still being quite extravagant in our use of filters, we make the following progress on the question from page 202 of [8] of whether every regular space is a dense subspace of some regular Baire space. We use the terminology "normal" and "regular" also for spaces which are not $T_{1}[6$, pages 112 and 113, ef. also 2, p. 118 and 5, p. 97].

THEOREM 3. Every topological space which is normal and regular is a dense subspace of some regular $\alpha$-favorable space.

Proof. Let $X$ be a normal space and let $F$ be the set of all maximal free regular filters on $X$. Since $X$ is normal it is quasiregular. Then any strategy $f$ for $\alpha$ in $G\left(X_{F}\right)$ for which $f$ is into $\mathscr{B}(X, F)$ and for which $f$ uses the quasi-regularity of $X$ with each move is a winning strategy for $\alpha$. In particular for a winning strategy $f$ for $\alpha$ in $G\left(X_{F}\right)$ choose for each nonempty open $W$ in $X_{F}$ a value $f(W)=V^{*}$ where for some $U^{*} \in \mathscr{B}(X, F), U^{*} \subset W, \bar{V} \subset U$ in $X, V \neq \phi$, and $V$ is open in $X$. The successive moves by $\alpha$ in a play of $G\left(X_{F}\right)$ in which $\alpha$ uses the strategy $f$ would be a nested sequence $\left\{V_{i}^{*}\right\}_{i \in \omega}$ of nonempty open sets $V_{i}^{*} \in \mathscr{B}(X, F)$ such that $\bar{V}_{i+1} \subset V_{i}$ in $X$. If we assume $\beta$ wins the play then $\bigcap_{i \in \omega} V_{i}=\phi$ and a maximal free regular filter that includes $\left\{V_{i} \mid i \in \omega\right\}$ would be a point in every term of the play contradicting the assumption that the play is a win for $\beta$.

To show that $X_{F}$ is regular we first prove that a quasi-regularity relationship between open sets of $X$ is inherited by the corresponding open sets in $X_{F}$. Let $U$ and $V$ be open sets of $X$ such that $\bar{V} \subset U$ and we will show that $\overline{V^{*}} \subset U^{*}$ in $X_{F}$. If $x \in X \cap \overline{V^{*}}$ then $x \in \bar{V} \subset$ $U$ in $X$ and $x \in U^{*} \supset U$ as required. Suppose, for proof by contradiction, that $x \in F \cap \overline{V^{*}}$ and $x \notin U^{*}$. Then for any set $W$ in the filter $x, W \cap V \neq \phi$ and $W \not \subset U$. Since $X$ is normal there is a sequence $\left\{R_{i}\right\}_{i \in \omega}$ of open sets of $X$ such that $\bar{V} \subset R_{i} \subset U$ in $X$ and $\bar{R}_{i+1} \subset R_{i}$ 
in $X$ for $i \in \omega$. Since $x$ is a regular filter there is a sequence $\left\{W_{i}\right\}_{i \in \omega}$ such that $W_{i} \in x$ and $\bar{W}_{i+1} \subset W_{i}$ in $X$ for $i \in \omega$. The set $\mathscr{H}=$ $x \cup\left\{R_{i} \cap W_{i} \mid i \in \omega\right\}$ is strictly larger than $x$, has the finite intersection property, has empty intersection, and for any $M \in \mathscr{H}$ there is $N \in$ $\mathscr{H}$ such that $\bar{N} \subset M$. This contradicts the assumption that $x$ is a maximal free regular filter and concludes our demonstration that $\overline{V^{*}} \subset U^{*}$ in $X_{F}$. For any $x \in U^{*} \in \mathscr{B}(X, F)$ there is $V$ open in $X$ such that $\bar{V} \subset U$ in $X$ and $x \in V^{*}$ (this uses the defining property of regular filter if $x$ is a filter which contains $U$ and it uses regularity of $X$ if $x \in U$ ). Since we have shown that in this case also $\overline{V^{*}} \subset$ $U^{*}$ we may conclude that $X_{F}$ is regular.

\section{REFERENCES}

1. J. M. Aarts and D. J. Lutzer, Pseudo-completeness and the product of Baire spaces, Pacific J. Math., 48 (1973), 1-10.

2. R. Brown, Elements of Modern Topology, McGraw-Hill, London, 1968.

3. G. Choquet, Lectures on analysis. Vol. I: Integration and Topological Vector Spaces, Benjamin, New York, 1969.

4. P. Fletcher and W. R. Lindgren, A note on spaces of second category, Archiv der Math., 24 (1973), 186-187.

5. M. C. Gemignani, Elementary Topology, Addison-Wesley, Reading, 1972.

6. J. L. Kelley, General Topology, D. Van Nostrand Company, Inc., New York, 1955.

7. M. R. Krom, Cartesian products of metric Baire spaces, Proc. Amer. Math. Soc., 42 (1974), 588-594.

8. R. A. McCoy, A Baire spacc extension, Proc. Amer. Math. Soc., 33 (1972), 199-202.

9. - A filter characterization of regular Baire spaces, Proc. Amer. Math. Soc., 40 (1973), 268-270.

10. J. C. Oxtoby, Cartesian products of Baire spaces, Fund. Math., 49 (1961), 157-166.

11. - Measure and Category, Springer-Verlag, New York, 1971.

12. J. R. Porter and C. Votaw, $S(\alpha)$ spaces and regular Hausdorff extensions, Pacific J. Math., 45 (1973), 327-345.

13. F. D. Tall, An alternative to the continuum hypothesis and its uses in general topology, Notices Amer. Math. Soc., 20 (1973).

14. H. E. White, Topological spaces that are $\alpha$-favorable for a player with perfect information, Notices Amer. Math. Soc., 20 (1973).

Received July 16, 1974 and in revised form September 12, 1974.

UNIVERSity OF CALIFORNIA, DAVIS 



\section{PACIFIC JOURNAL OF MATHEMATICS}

\section{EDITORS}

RICHARD ARENS (Managing Editor)

University of California

Los Angeles, California 90024

\section{R. A. Beaumont \\ University of Washington \\ Seattle, Washington 98105}

\section{J. DugundJI}

Department of Mathematics

University of Southern California

Los Angeles, California 90007

D. Gilbarg and J. Milgram

Stanford University

Stanford, California 94305

\section{ASSOCIATE EDITORS}
E. F. BECKENBACH
B. H. NeumanN
F. WOLF
K. YOSHIDA

\section{SUPPORTING INSTITUTIONS}

UNIVERSITY OF BRITISH COLUMBIA
CALIFORNIA INSTITUTE OF TECHNOLOGY
UNIVERSITY OF CALIFORNIA
MONTANA STATE UNIVERSITY
UNIVERSITY OF NEVADA
NEW MEXICO STATE UNIVERSITY
OREGON STATE UNIVERSITY
UNIVERSITY OF OREGON
OSAKA UNIVERSITY

UNIVERSITY OF BRITISH COLUMBIA

UNIVERSITY OF CALIFORNIA

MONTANA STATE UNIVERSITY

NEW MEXICO STATE UNIVERSITY

OREGON STATE UNIVERSITY

OSAKA UNIVERSITY

\author{
UNIVERSITY OF SOUTHERN CALIFORNIA \\ STANFORD UNIVERSITY \\ UNIVERSITY OF TOKYO \\ UNIVERSITY OF UTAH \\ WASHINGTON STATE UNIVERSITY \\ UNIVERSITY OF WASHINGTON \\ AMERICAN MATHEMATICAL SOCIETY \\ NAVAL WEAPONS CENTER
}

The Supporting Institutions listed above contribute to the cost of publication of this Journal, but they are not owners or publishers and have no responsibility for its content or policies.

Mathematical papers intended for publication in the Pacific Journal of Mathematics should be in typed form or offset-reproduced, (not dittoed), double spaced with large margins. Underline Greek letters in red, German in green, and script in blue. The first paragraph or two must be capable of being used separately as a synopsis of the entire paper. Items of the bibliography should not be cited there unless absolutely necessary, in which case they must be identified by author and Journal, rather than by item number. Manuscripts, in triplicate, may be sent to any one of the editors. Please classify according to the scheme of Math. Reviews, Index to Vol. 39. All other communications should be addressed to the managing editor, or Elaine Barth, University of California, Los Angeles, California, 90024.

The Pacific Journal of Mathematics expects the author's institution to pay page charges, and reserves the right to delay publication for nonpayment of charges in case of financial emergency.

100 reprints are provided free for each article, only if page charges have been substantially paid. Additional copies may be obtained at cost in multiples of 50 .

The Pacific Journal of Mathematics is issued monthly as of January 1966. Regular subscription rate: $\$ 72.00$ a year (6 Vols., 12 issues). Special rate: $\$ 36.00$ a year to individual members of supporting institutions.

Subscriptions, orders for back numbers, and changes of address should be sent to Pacific Journal of Mathematics, 103 Highland Boulevard, Berkeley, California, 94708.

PUBLISHED BY PACIFIC JOURNAL OF MATHEMATICS, A NON-PROFIT CORPORATION

Printed at Kokusai Bunken Insatsusha (International Academic Printing Co., Ltd.), 270, 3-chome Totsuka-cho, Shinjuku-ku, Tokyo 160, Japan.

Copyright (C) 1973 by Pacific Journal of Mathematics Manufactured and first issued in Japan 


\section{Pacific Journal of Mathematics \\ Vol. 55, No. $2 \quad$ October, 1974}

Walter Allegretto, On the equivalence of two types of oscillation for elliptic

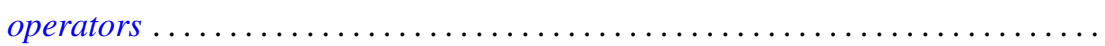

Edward Arthur Bertram, A density theorem on the number of conjugacy classes in

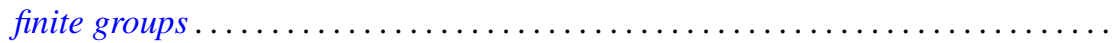

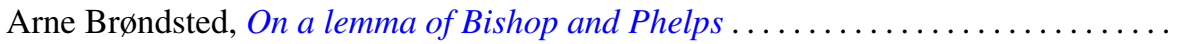

Jacob Burbea, Total positivity and reproducing kernels ..................

Ed Dubinsky, Linear Pincherle sequences . . . . . . . . . . . . . . . . . .

Benny Dan Evans, Cyclic amalgamations of residually finite groups .............

361

Barry J. Gardner and Patrick Noble Stewart, A "going down" theorem for certain

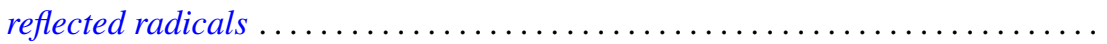

381

Jonathan Light Gross and Thomas William Tucker, Quotients of complete graphs:

revisiting the Heawood map-coloring problem ....................

Sav Roman Harasymiv, Groups of matrices acting on distribution spaces .........

Robert Winship Heath and David John Lutzer, Dugundji extension theorems for

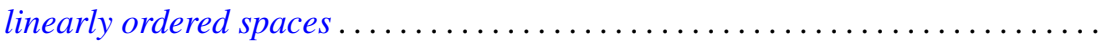

Chung-Wu Ho, Deforming p. l. homeomorphisms on a convex polygonal

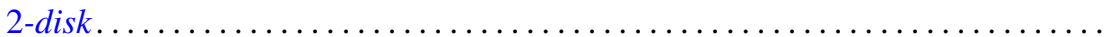

Richard Earl Hodel, Metrizability of topological spaces .................

Wilfried Imrich and Mark E. Watkins, On graphical regular representations of

cyclic extensions of groups .......................... 461

Jozef Krasinkiewicz, Remark on mappings not raising dimension of curves ..... . 479

Melven Robert Krom, Infinite games and special Baire space extensions . . . . . . 483

S. Leela, Stability of measure differential equations . . . . . . . . . . . . . . . . 489

M. H. Lim, Linear transformations on symmetric spaces . . . . . . . . . . . . . . . 499

Teng-Sun Liu, Arnoud C. M. van Rooij and Ju-Kwei Wang, On some group algebra modules related to Wiener's algebra $M_{1} \ldots \ldots \ldots \ldots \ldots \ldots \ldots \ldots \ldots \ldots \ldots$

Dale Wayne Myers, The back-and-forth isomorphism construction ............ 521

Donovan Harold Van Osdol, Extensions of sheaves of commutative algebras by

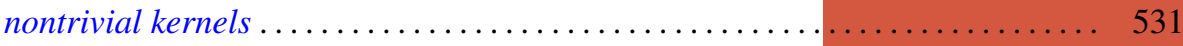

Alan Rahilly, Generalized Hall planes of even order ................... 543

Joylyn Newberry Reed, On completeness and semicompleteness of first countable

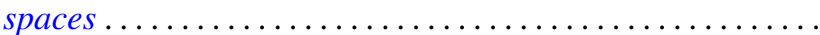

Alan Schwartz, Generalized convolutions and positive definite functions associated

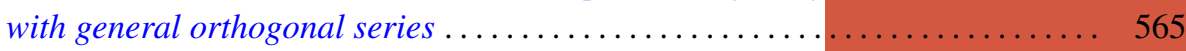

Thomas Jerome Scott, Monotonic permutations of chains . . . . . . . . . . . 583

Eivind Stensholt, An application of Steinberg's construction of twisted groups .... 595

Yasuji Takeuchi, On strongly radicial extensions . . . . ................. 619

William P. Ziemer, Some remarks on harmonic measure in space . . . . . . . . . . 629

John Grant, Corrections to: “Automorphisms definable by formulas” . . . . . . . . 639

Peter Michael Rosenthal, Corrections to: "On an inversion for the general

Mehler-Fock transform pair" ......................... 640

Carl Clifton Faith, Corrections to: "When are proper cyclics injective” . . . . . . 640 